\title{
Decline in Hepatitis E Virus Antibody Prevalence in Southeastern Germany, 1996-2011
}

\author{
Jürgen J. Wenzel, ${ }^{1}$ Mathias Sichler, ${ }^{1}$ Mathias Schemmerer, ${ }^{1}$ Gundula Behrens, ${ }^{2}$ \\ Michael F. Leitzmann, ${ }^{2}$ and Wolfgang Jilg $^{1}$
}

In the past decade, an increasing frequency of acute hepatitis $\mathrm{E}$ was noted in Germany and other European countries. Moreover, a high prevalence $(17 \%)$ of hepatitis $\mathrm{E}$ virus (HEV) immunoglobulin G antibodies (anti-HEV) was recently found in the adult German population. Although this suggests an emerging pathogen, reports from other countries gave hints to a completely new aspect: a possible decrease in anti-HEV prevalence during the last decades. To investigate the time trends of hepatitis $\mathrm{E}$ in southeastern Germany, we performed anti-HEV testing in sera taken from adults in 1996 and 2011. Surplus serum specimens stored during routine operations of our diagnostic laboratory were used. The sample comprised two sets of 1,092 sera taken in 1996 and 2011, each with 182 specimens in six age groups from 20-79 years. Testing was performed using an HEV IgG enzyme immunoassay (EIA, Axiom Diagnostics), and the recomLine HEV IgG immunoblot (Mikrogen). A significant difference in anti-HEV prevalence was observed between the two groups: $50.7 \%$ of individuals tested positive in the 1996 group as compared to $34.3 \%$ in 2011 (EIA, $P<0.001$ ). Results by immunoblot analysis were $20.5 \%(1996)$ versus $14.5 \%$ (2011), $P<0.001$. Differences were found in all age groups and were more pronounced for the 20-39-year age group. Conclusion: The prevalence of anti-HEV has decreased significantly in the past decades in southeastern Germany. The phenomenon of HEV being an emerging pathogen is thus most probably due to an increasing awareness of the disease. (HEPATOLOGY 2014;60:1180-1186)

$\mathrm{H}$ epatitis E virus (HEV) is a small, nonenveloped RNA virus with a genome size of $\sim 7.2$ $\mathrm{kb} . \mathrm{HEV}$ is a member of the genus Hepevirus, family Hepeviridae. The virus was first postulated in 1980 as a causative agent of enterically transmitted non-A, non-B hepatitis in India and was identified 3 years later. ${ }^{1}$ Subsequent analyses of viral genomes led to the identification of four mammalian HEV genotypes with distinct geographic distributions. Genotype 1 is a major cause of infectious hepatitis transmitted by the fecal-oral route in Asia and Africa. ${ }^{2}$ Consequently, acute hepatitis $\mathrm{E}$ has previously been considered an exclusively travel-associated disease acquired in one of these geographic areas. However, evidence has accumulated that HEV infection also occurs among individuals in industrialized countries with no history of travel to HEV-endemic areas. ${ }^{3-5}$ These autochtho- nous cases are caused mainly by HEV genotype 3 (and in rare instances genotype 4) strains. ${ }^{3,5-8}$ Because genotype 3 isolates from human cases were almost identical to strains detected in swine and wild boars, zoonotic sources of infection are suspected. ${ }^{7,9,10} \mathrm{HEV}$ genotype 3 infections are usually asymptomatic and hence remain undiagnosed. Only a very small minority of cases, probably less than $1 \%,{ }^{11}$ show symptoms of acute viral hepatitis. However, several chronic cases have been reported in transplant recipients. ${ }^{12-14}$

In Germany, as in other European countries, ${ }^{15,16}$ a rapidly increasing frequency of acute hepatitis $\mathrm{E}$ has been noted in the past decade, with 32 notified cases in $2003^{17}$ rising to 454 cases in $2013 .{ }^{18}$ The majority of strains isolated from patients with acute hepatitis $\mathrm{E}$ in Germany were genotype 3. 5,7,19 Despite relatively low numbers of acute cases, surprisingly high anti-

Abbreviations: Anti-HEV, hepatitis E virus immunoglobulin G antibodies; EIA, enzyme immunoassay; FOI, force of infection; HEV, hepatitis Evirus; LoD, lower limit of detection; NIBSC, National Institute for Biological Standards and Control; OD, optical density; SCR, signal-to-cutoff ratio; WHO, World Health Organization.

From the ${ }^{1}$ Institute of Clinical Microbiology and Hygiene, University Medical Center Regensburg, Regensburg, Germany; ${ }^{2}$ Department of Epidemiology and Preventive Medicine, University of Regensburg, Regensburg, Germany.

Received March 21, 2014; accepted May 26, 2014. 
HEV prevalence estimates were reported in different European populations. ${ }^{20,21}$ In contrast to other studies, which reported lower rates, these studies applied antiHEV detection assays with higher sensitivity for the prevalent genotype 3 strains.

The anti-HEV prevalence was recently determined in a large representative sample of the German adult population. The overall prevalence as determined by immunoblot was $16.8 \%$, increased with age, and leveled off at $>60$ years of age. ${ }^{11}$ In summary, these characteristics all imply that $\mathrm{HEV}$ is an emerging pathogen. In sharp contrast to this notion, two recent reports from England and Denmark gave hints of a completely new aspect: a possible decrease in antiHEV prevalence during the past decades. ${ }^{22,23}$ Referring to these publications, the purpose of our work was to investigate these hints in more detail by conducting a study which is 1) appropriately powered, 2) geographically focused, and 3) based on comparable study participants. The principal question was whether there is a significant time trend of hepatitis E seroprevalence in Southeastern Germany. To answer this question, sera taken from adults in 1996 and 2011 were analyzed for the presence of anti-HEV.

\section{Materials and Methods}

Sample Composition and Study Design. A total of 2,184 specimens were included in the study. In all, $1,092(50 \%)$ samples were collected in 1996 and 1,092 were collected in 2011. In the 1996 sample set, $96.3 \%(1,052 / 1,092)$ were from the federal state of Bavaria in southeastern Germany. A minority of $3.7 \%$ $(40 / 1,092)$ were from other German federal states or could not be assigned to a distinct geographic location. The corresponding numbers for the 2011 sample set are $96.4 \%(1,053 / 1,092)$ and $3.6 \%(39 / 1,092)$, respectively. The specimen sets comprised six age groups for 1996 and 2011, respectively, each consisting of 182 samples. An equal gender distribution was chosen within each of the 12 age groups (Fig. 1).

Sample Collection. All serum samples were obtained as part of the daily routine operations of our diagnostic laboratory in 1996 and 2011, and only stored surplus serum was used for this study. Samples were randomly chosen from all available specimens of a certain age group with no preselection as to the patients' clinical characteristics. If a specimen was included in the 1996 sample set, specimens available from the same person in 2011 were excluded. All sera were stored at $-20^{\circ} \mathrm{C}$ prior to testing.

Reference Reagents. The WHO reference reagent (NIBSC code: 95/584) for antibodies to HEV was used as a biological standard for antibody concentration. ${ }^{24}$ Moreover, a second reference reagent containing antibodies against HEV genotype 3 was analyzed. ${ }^{7}$ Details about the reference reagents and experimental setup are presented in the Supporting Information.

Laboratory Analysis. Serum samples were tested for the presence of anti-HEV using two commercially available immunoassays: the Axiom Diagnostics (Worms, Germany) HEV IgG EIA (developed by Wantai, Beijing, China), and the Mikrogen recomLine HEV IgG immunoblot (Mikrogen, Neuried, Germany), denoted hereafter as EIA and immunoblot, respectively. A cutoff optical density (OD) for the EIA was determined according to the manufacturer by adding 0.16 to the arithmetic mean of the negative control ODs. For each measurement, a signal-to-cutoff ratio (SCR) was calculated by dividing the respective OD by the cutoff OD. A modified EIA scoring system was applied with a negative result being recorded for SCRs of $\leq 1.1$, and a positive result for SCRs of $>1.1$. The quantitative interpretation of SCR values was possible within the linear range of the assay (Supporting Information Fig. 1). The immunoblot is based on seven recombinant antigens of HEV genotypes 1 and 3. Stained test strips were scanned with the semiautomated recomScan apparatus and results were calculated with the respective analysis software (Mikrogen). Each antigen band with an intensity greater than or equal to the cutoff band was assigned a predefined point value. The final results were classified into two categories: negative (defined as $\leq 2$ points) and positive (defined as $\geq 3$ points). To achieve better comparability of the analytical performance experiments, SCR values were also calculated for immunoblot measurements by dividing the mean ODs of reactive antigen bands by the density of the respective cutoff band.

Address reprint requests to: Jürgen J. Wenzel, M.D., Institute of Clinical Microbiology and Hygiene, University Medical Center Regensburg, Franz-Josef-StraussAllee 11, 93053 Regensburg, Germany. E-mail: juergen.wenzel@ukr.de; fax: +49 (941) 9446402.

Copyright $(2014$ by the American Association for the Study of Liver Diseases.

View this article online at wileyonlinelibrary.com.

DOI 10.1002/hep. 27244

Potential conflict of interest: Dr. Wenzel received payment for lectures by Mikrogen. Dr. Jilg received payment for lectures by Mikrogen. 


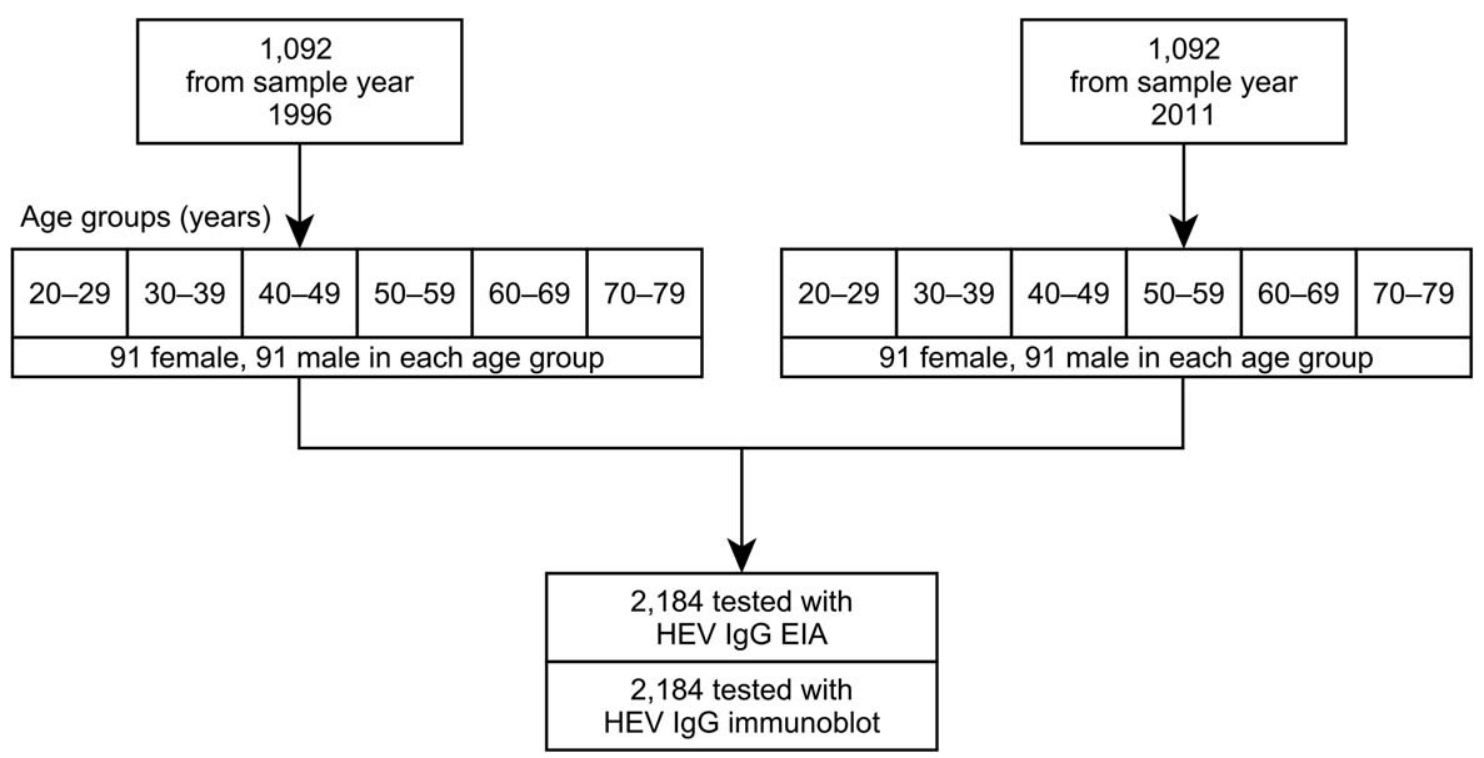

Fig. 1. Study design and sample composition. HEV, hepatitis E virus; IgG, immunoglobulin G; EIA, enzyme immunoassay.

Statistical Analysis. To determine a suitable sample size, the assumption was made that the anti-HEV prevalence in 1996 differed by at least 5\% from the prevalence in 2011. We estimated that 1,092 subjects were necessary per sample year to detect a significant effect with $95 \%$ confidence and $85 \%$ statistical power. Sample size and power calculations were performed with the software $\mathrm{G}^{*}$ Power $3 .^{25}$ Random sampling of specimens was carried out with the sample() function implemented by the $\mathrm{R}$ programming language ( $\mathrm{R}$ Foundation for Statistical Computing). Pearson's $\chi^{2}$ test was used to evaluate differences between the antiHEV prevalence rates in 1996 and 2011. Data processing and statistical analysis were performed with SPSS v. 20.0 (IBM, Ehingen, Germany). A statistically significant difference was defined as $P<0.05$.

\section{Results}

Anti-HEV Prevalence in 1996 and 2011. In both assays, a significantly higher anti-HEV prevalence was seen in the group sampled in 1996 than in the individuals from 2011. By EIA, 50.7\% $(554 / 1,092)$ were positive for anti-HEV in 1996 and 34.3\% (375/1,092) were positive in $2011(P<0.001)$. The corresponding values obtained by the immunoblot assay were $20.5 \%$ $(224 / 1,092)$ and $14.5 \%(158 / 1,092 ; P<0.001)$ (Fig. $2 \mathrm{~A})$. Although the results by the two methods differed considerably, in both groups sera tested positive by immunoblot were an almost complete subset of the positive sera by EIA (Fig. 2B): $96.4 \%$ and 94.9\%, respectively, of immunoblot-positive sera tested in 1996 and 2011 were also positive with EIA, whereas only a small percentage of $3.6 \%$ and $5.1 \%$ were solely immunoblot-positive.

Analysis of Different Age Groups. Anti-HEV prevalence increased with age between 20 and 59 years in the 1996 group and between 30 and 69 years in the 2011 group (Fig. 3). Gender-specific differences were not observed (data not shown). Comparison of the EIA prevalence estimates by age group between 1996 and 2011 showed significantly lower rates in 2011 for the age groups 20-29 years $(P=0.001)$, 3039 years $(P<0.001), 40-49$ years $(P<0.001)$, and 50 59 years $(P=0.002)$. Differences were not significant in the age groups $60-69$ years $(P=0.345)$ and $70-79$ years $(P=0.173$; Fig. $3 A)$. Results obtained with the immunoblot assay were almost identical to the EIA results but on a considerably lower level. Accordingly, significant differences were found in the age groups of 20-49 years: $20-29$ years $(P=0.022), 30-39$ years $(P<0.001), \quad 40-49$ years $(P=0.031), \quad 50-59$ years $(P=0.307), 60-69$ years $(P=0.218)$, and $70-79$ years $(\mathrm{P}=0.197$; Fig. 3B $)$. The anti-HEV prevalence in the two 60-69 year age groups as assessed by immunoblot showed-different from all other comparisons-a lower value in $1996(P=0.218)$. The 60-69 year age group also showed a lower value in the EIA assay than the older and younger age groups, causing a dip in the curve of the prevalence values (Fig. 3A). The most pronounced absolute difference in the prevalence rates between 1996 and 2011 was found in the age group 
A

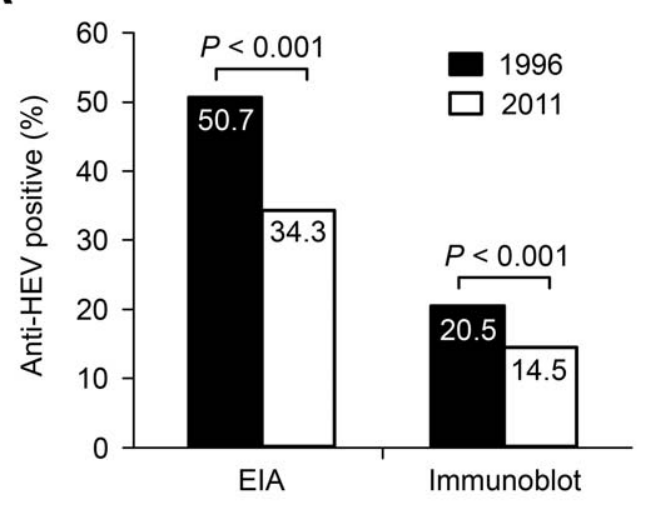

B

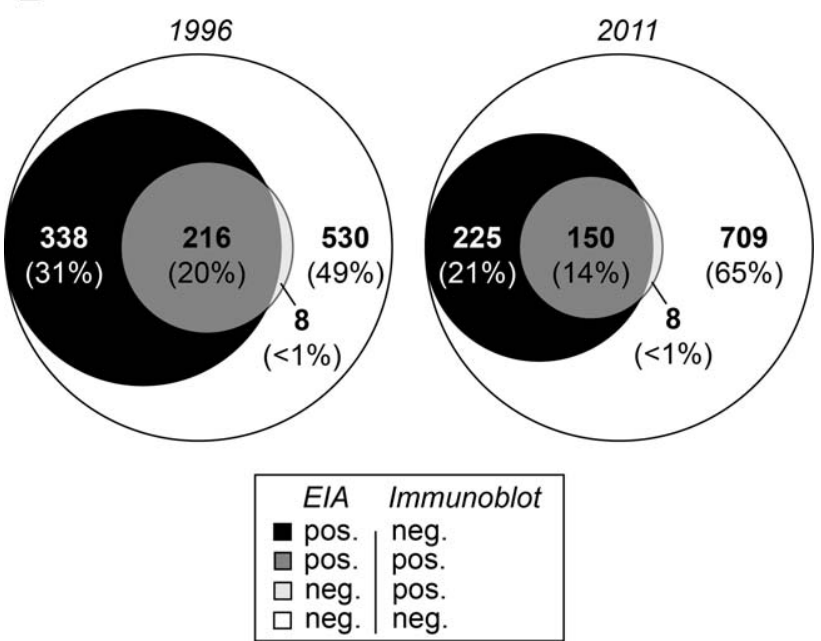

Fig. 2. (A) Anti-HEV prevalence in 1996 and 2011. Results were generated with the Axiom Diagnostics HEV IgG enzyme immunoassay (EIA), and the Mikrogen recomLine HEV IgG assay (immunoblot). Pearson's $\chi^{2}$ test was used to evaluate differences between the anti-HEV prevalences in 1996 and 2011. (B) Schematic distribution of results generated with the EIA and with the immunoblot. Absolute numbers and calculated ratios (\%) are indicated. Pos., positive; neg., negative.

30-39 years: a 36.3\% lower value when measured by EIA $(P<0.001)$ and a $14.9 \%$ lower value when measured by immunoblot $(P<0.001)$. The difference decreased continuously between 30 and 59 years. Comparing the EIA profiles of 30 acute hepatitis E cases $^{7}$ with anti-HEV positive samples from an exemplary age-group in the current study showed that acute or recent cases show a single high peak of anti-HEV reactivity, whereas the groups analyzed in the current study show a broader distribution of anti-HEV SCRs (Supporting Information Fig. 2A,B).

Birth Cohort Analysis. Anti-HEV prevalences of the 1996 and 2011 groups were also analyzed according to the birth cohort. Both groups included individuals born between 1930 and 1979. Surprisingly, in all 10-year birth cohorts, individuals sampled in 2011 showed lower anti-HEV prevalences than those sampled in 1996 (Fig. 4, Supporting Information Table). Whereas these differences were only marginal in the two youngest groups (born 1960-1969 and 1970-1979), they became rather evident in individuals born between 1930 and 1959, reaching statistical significance in the 1940-1949 birth cohort $(P=0.02)$, suggesting a loss of specific antibodies over time. Analyzing the distribution of SCR values by EIA allowed us to approximate the proportion of acute or recent infections empirically, defined as samples with SCR $\geq 16$ : all birth cohorts had a lower rate of acute or recent infections in 2011 as compared to 1996 (Supporting Information Fig. 2C).

Analytical Performance Validation of the Immunoassays. The analytical performance of the antiHEV detection assays was evaluated by analyzing a
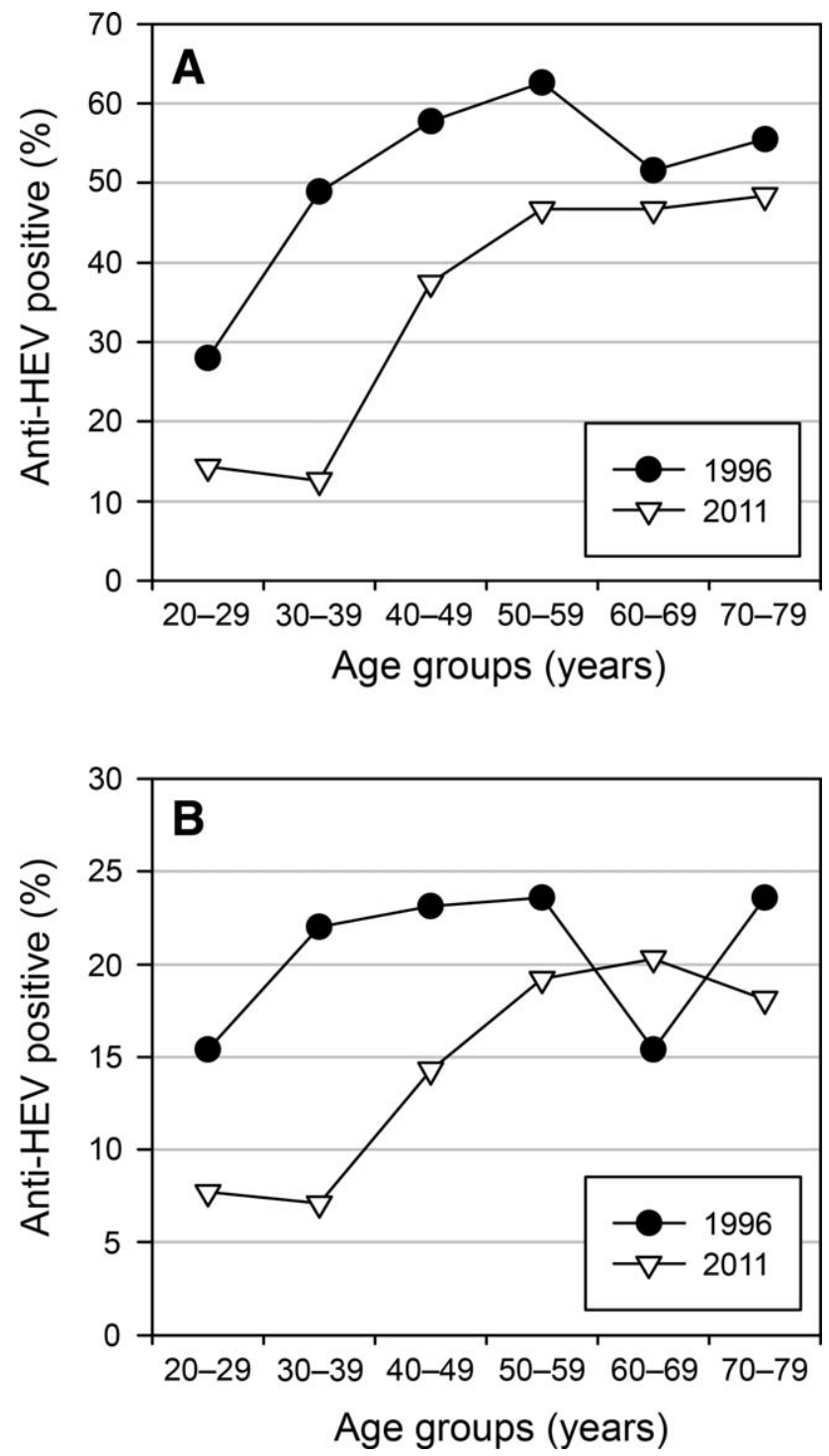

Fig. 3. Anti-HEV prevalence shown by age group. (A) Results obtained by ElA. (B) Results obtained by immunoblot analysis. 


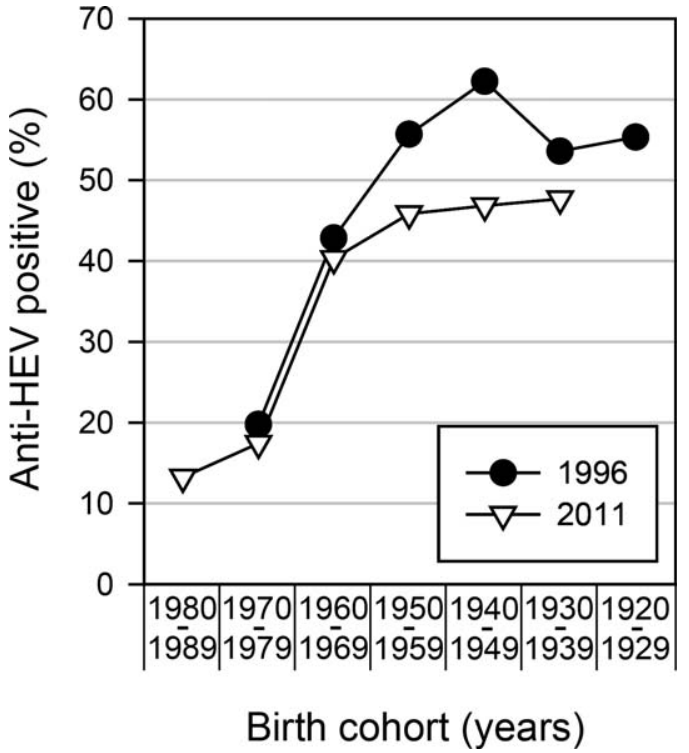

Fig. 4. Anti-HEV prevalence shown by birth cohort. Results were obtained by EIA.

dilution series of the two reference reagents. In summary, both assays proved to be capable of sensitively detecting anti-HEV in dilutions of both reference materials. The lower limit of detection (LoD, defined as the lowest anti-HEV concentration with a positive test result) for the World Health Organization (WHO) reagent was determined at $0.39 \mathrm{U} / \mathrm{mL}$ with the EIA and $0.78 \mathrm{U} / \mathrm{mL}$ with the immunoblot assay. The corresponding values for the genotype 3 reference were $0.39 \mathrm{U} / \mathrm{mL}$ (EIA) and $1.57 \mathrm{U} / \mathrm{mL}$ (immunoblot). Detailed results of the analytical performance validation are presented in Supporting Information Fig. 1.

\section{Discussion}

In this study we examined whether the prevalence of anti-HEV has changed over time in southeastern Germany. To address this issue, we compared sera taken from adults in 1996 and 2011 using two different anti-HEV detection assays. Our results show that 1) overall anti-HEV prevalence has declined significantly over time; 2) the decrease in prevalence is most prominent in the 20-49 year age group; and 3) this effect is evident regardless of the detection assay used.

Our findings are substantiated by an assay performance validation, which shows that both assays are suitable for detecting anti-HEV directed against the relevant $\mathrm{HEV}$ genotypes. The study was designed to detect a $5 \%$ difference in anti-HEV prevalence with $85 \%$ statistical power and a $5 \%$ probability of error.
Statistical analysis demonstrated an overall decline in anti-HEV prevalence of $15.7 \%$ (EIA) and 6\% (immunoblot) with $P<0.001$. This finding is supported by the fact that significant decreases in anti-HEV prevalence were also found in the analyses for the younger age groups and with data generated by both detection assays. However, no statistically significant difference was found in the 60-69 and 70-79 year age groups.

Surprisingly, birth cohort analysis showed in all groups higher prevalences in sera taken in 1996 versus sera of 2011. Differences were marginal in those born in 1970-79 and 1960-69, but higher in the other groups and even statistically significant in the 19401949 birth cohort. A likely interpretation is the occurrence of waning immunity, manifesting itself as a decreasing anti-HEV concentration after infection over time. $^{26}$ Thus, the observed difference in the total antiHEV prevalence would be the result of negative seroconversions due to waning immunity, considerably outnumbering positive seroconversions due to novel $\mathrm{HEV}$ infections (and probably reinfections) at the same time. ${ }^{26,27}$ This interpretation is supported by the SCR distribution analysis, where acute cases ${ }^{7}$ show a single high peak of anti-HEV reactivity, whereas the groups analyzed in the present study showed a broader SCR distribution. A limitation of our study is that further investigation of this aspect is not possible due to the lack of consecutively drawn serum samples. Mathematical models aimed at estimating the incidence and force of infection (FOI) regularly assume constant seropositivity after infection. ${ }^{11}$ Since we cannot separately characterize the extent of positive versus negative seroconversions, we did not apply mathematical modeling. Nevertheless, the high prevalence in subjects born before $\sim 1960$ implies that these individuals lived through a period with increased risk of HEV transmission, but this risk has been decreasing since and is currently at a lower level. The lower ratio of putative acute or recent infections observed in the 2011 sample supports this conclusion.

Previous studies have found high anti-HEV prevalences in Germany and other industrialized countries, which were previously regarded as nonendemic for HEV. ${ }^{11,19,20}$ The anti-HEV prevalence for our 2011 sample fits well with these previous reports. ${ }^{11,19}$ It is a limitation of our study that we could only test leftover sera of a confined geographic origin. However, a nationwide study found no differences between antiHEV prevalence rates in different geographical areas within Germany. ${ }^{11}$ Although these data do not completely rule out risk variations in certain age groups or localizations (e.g., in the older population due to the 
former separation of the country), it is yet tempting to speculate that our results are representative for all of Germany.

Recently, evidence has accumulated that different anti-HEV detection assays strongly affect anti-HEV prevalence estimates. ${ }^{19,21}$ Hence, it is a technical prerequisite that assays with high sensitivity for HEV genotype 3 are used for prevalence studies in Europe. We demonstrated in this work that both detection assays used fulfill these requirements. Moreover, the comparability of results to other studies is warranted by using the same immunoblot assay as previously. ${ }^{11,19}$ The distribution of results shows that the positives by immunoblot are almost a complete subset of the positives by EIA. This can be explained by the higher sensitivity of the EIA. Random results due to a lack of specificity would not show such a pattern. ${ }^{19}$

Two previous studies monitored anti-HEV prevalence over time. Unexpectedly, both studies found hints of effectively declining anti-HEV prevalences. However, both studies were limited for several reasons. Ijaz et al. determined the anti-HEV prevalence in 2,731 samples from England and Wales. ${ }^{22}$ The authors used a sensitive HEV IgG EIA, but-different from our results-found no significantly different anti-HEV prevalences in the 1991 (13.0\%) and 2004 (13.5\%) sample collections. Cautious interpretation of these results is necessary because the geographical origin of the samples was from two whole countries. Also, samples were unevenly matched between the two sampling years. A sampling effect might thus have influenced the overall result or regional differences might have been overlooked. Ijaz et al. ${ }^{22}$ found an overall association between increase in prevalence and increase in age. The authors speculate that the risk of acquiring HEV infection in England and Wales rose sharply during the mid- $20^{\text {th }}$ century, but then decreased at the end of the 1950s and currently remains low. Our results from the birth cohort analysis fit well with this model. The second study by Christensen et al. compared the anti-HEV prevalence in Denmark by analyzing two sets of sera taken in $1983(\mathrm{n}=169)$ and 2003 $(\mathrm{n}=461){ }^{23}$ The authors found a significant decrease in anti-HEV prevalence from $32.9 \%$ to $20.6 \%$. However, cautious interpretation is advisable due to the small sample size, uneven sample distribution (according to sample years), and the divergent sample origin (blood donors, farmers, and prisoners). In order to avoid these potential drawbacks, our study comprised a significantly larger sample size and was based on two geographically focused and highly comparable study collectives. Our results suggest that the rate of HEV exposure has decreased considerably in the past. This notion is supported by a study on the evolutionary history and population dynamics of $\mathrm{HEV}$ : based on in silico modeling, the authors propose that genotypes 3 and 4 have experienced an increase in population size in the first half of the $20^{\text {th }}$ century, followed by a decline of unknown cause around $1990 .^{28}$ Another study from Japan points in the same direction. However, the latter model anticipates an increase in HEV genotype 3 population size in Japan around 1960-1970, followed by a decline around $2000 .{ }^{29}$

Some important questions remain: why is a large portion of the German population anti-HEV positive? What is the reason for the declining prevalence in the last decades? There is now a growing body of evidence suggesting that autochthonous HEV infections are transmitted by ingestion of contaminated pork products. $^{7,30-34}$ If this is the major mode of transmission, then there must also have been a significant reduction in alimentary exposure to $\mathrm{HEV}$ in the past decades, presumably resulting in the interruption of HEV transmissions. We can only speculate that improvements in the food industry are the underlying reason, since the per capita pork intake was relatively stable between 1990 and 2010 in Germany.

In summary, our study shows that the anti-HEV prevalence has significantly decreased in the past decades in southeastern Germany. The risk of HEV infection was likely higher in the first half of the $20^{\text {th }}$ century and has been decreasing in the past decades. Currently observed increasing numbers of acute hepatitis $\mathrm{E}$ cases should be therefore interpreted as an artifact probably caused by a higher awareness of the disease.

Acknowledgment: We thank Barbara Schmidt for thoughtful review of the article and continuous support.

\section{References}

1. Balayan MS, Andjaparidze AG, Savinskaya SS, Ketiladze ES, Braginsky DM, Savinov AP, et al. Evidence for a virus in non-A, non-B hepatitis transmitted via the fecal-oral route. Intervirology 1983;20:23-31.

2. Purcell RH, Emerson SU. Hepatitis E: an emerging awareness of an old disease. J Hepatol 2008;48:494-503.

3. Brost S, Wenzel JJ, Ganten TM, Filser M, Flechtenmacher C, Boehm $\mathrm{S}$, et al. Sporadic cases of acute autochthonous hepatitis $\mathrm{E}$ virus infection in southwest Germany. J Clin Virol 2010;47:89-92.

4. Schlauder GG, Dawson GJ, Erker JC, Kwo PY, Knigge MF, Smalley $\mathrm{DL}$, et al. The sequence and phylogenetic analysis of a novel hepatitis $\mathrm{E}$ virus isolated from a patient with acute hepatitis reported in the United States. J Gen Virol 1998;79(Pt 3):447-456.

5. Wichmann O, Schimanski S, Koch J, Kohler M, Rothe C, Plentz A, et al. Phylogenetic and case-control study on hepatitis $\mathrm{E}$ virus infection in Germany. J Infect Dis 2008;198:1732-1741.

6. Preiss JC, Plentz A, Engelmann E, Schneider T, Jilg W, Zeitz M, et al. Autochthonous hepatitis $\mathrm{E}$ virus infection in Germany with 
sequence similarities to other European isolates. Infection 2006;34: 173-175.

7. Wenzel JJ, Preiss J, Schemmerer M, Huber B, Plentz A, Jilg W. Detection of hepatitis E virus (HEV) from porcine livers in Southeastern Germany and high sequence homology to human HEV isolates. J Clin Virol 2011;52:50-54.

8. Jeblaoui A, Haim-Boukobza S, Marchadier E, Mokhtari C, RoqueAfonso AM. Genotype 4 hepatitis E virus in France: an autochthonous infection with a more severe presentation. Clin Infect Dis 2013;57: e122-126.

9. Li TC, Chijiwa K, Sera N, Ishibashi T, Etoh Y, Shinohara Y, et al. Hepatitis E virus transmission from wild boar meat. Emerg Infect Dis 2005;11:1958-1960.

10. Tei S, Kitajima N, Takahashi K, Mishiro S. Zoonotic transmission of hepatitis E virus from deer to human beings. Lancet 2003;362:371373.

11. Faber MS, Wenzel JJ, Jilg W, Thamm M, Hohle M, Stark K. Hepatitis E virus seroprevalence among adults, Germany. Emerg Infect Dis 2012;18:1654-1657.

12. Hoerning A, Hegen B, Wingen AM, Cetiner M, Lainka E, Kathemann $S$, et al. Prevalence of hepatitis E virus infection in pediatric solid organ transplant recipients - a single-center experience. Pediatr Transplant 2012;16:742-747.

13. Kamar N, Selves J, Mansuy JM, Ouezzani L, Peron JM, Guitard J, et al. Hepatitis $\mathrm{E}$ virus and chronic hepatitis in organ-transplant recipients. N Engl J Med 2008;358:811-817.

14. Pischke S, Stiefel P, Franz B, Bremer B, Suneetha PV, Heim A, et al. Chronic hepatitis $\mathrm{E}$ in heart transplant recipients. Am J Transplant 2012;12:3128-3133.

15. Public Health England. Infectious diseases surveillance. Hepatitis E. Available at: http://www.hpa.org.uk/Topics/InfectiousDiseases/Infections AZ/HepatitisE/Surveillance/. Accessed 5 May 2014.

16. The French National Research Agency. Hepatitis E dynamics in related ecosystems: from pig farms and wastewaters to shellfish. Available at: http://www.agence-nationale-recherche.fr/en/anr-funded-project/?tx_lwm suivibilan_pi2[CODE]=ANR-10-CESA-0010. Accessed 5 May 2014.

17. Robert Koch-Institut. Infektionsepidemiologisches Jahrbuch für 2003. Available at: http://www.rki.de/DE/Content/Infekt/Jahrbuch/Jahrbuecher/ 2003.html. Accessed 5 May 2014.

18. Robert Koch-Institut. Infektionsepidemiologisches Bulletin 7/2014. Available at: https://www.rki.de/DE/Content/Infekt/EpidBull/Archiv/ 2014/Ausgaben/07_14.html. Accessed 5 May 2014.

19. Wenzel JJ, Preiss J, Schemmerer M, Huber B, Jilg W. Test performance characteristics of anti-HEV IgG assays strongly influence hepatitis E seroprevalence estimates. J Infect Dis 2013;207:497-500.

20. Bendall R, Ellis V, Ijaz S, Ali R, Dalton H. A comparison of two commercially available anti-HEV IgG kits and a re-evaluation of anti-HEV IgG seroprevalence data in developed countries. J Med Virol 2010;82: 799-805.

21. Mansuy JM, Bendall R, Legrand-Abravanel F, Saune K, Miedouge M, Ellis V, et al. Hepatitis E virus antibodies in blood donors, France. Emerg Infect Dis 2011;17:2309-2312.
22. Ijaz S, Vyse AJ, Morgan D, Pebody RG, Tedder RS, Brown D. Indigenous hepatitis $\mathrm{E}$ virus infection in England: more common than it seems. J Clin Virol 2009;44:272-276.

23. Christensen PB, Engle RE, Hjort C, Homburg KM, Vach W, Georgsen J, et al. Time trend of the prevalence of hepatitis $\mathrm{E}$ antibodies among farmers and blood donors: a potential zoonosis in Denmark. Clin Infect Dis 2008;47:1026-1031.

24. Ferguson M, Walker D, Mast E, Fields H. Report of a collaborative study to assess the suitability of a reference reagent for antibodies to hepatitis E virus. Biologicals 2002;30:43-48.

25. Faul F, Erdfelder E, Lang AG, Buchner A. G*Power 3: a flexible statistical power analysis program for the social, behavioral, and biomedical sciences. Behav Res Methods 2007;39:175-191.

26. Zhang J, Li SW, Wu T, Zhao Q, Ng MH, Xia NS. Hepatitis E virus: neutralizing sites, diagnosis, and protective immunity. Rev Med Virol 2012;22:339-349.

27. Bendall R, Ellis V, Ijaz S, Thurairajah P, Dalton HR. Serological response to hepatitis $\mathrm{E}$ virus genotype 3 infection: $\operatorname{IgG}$ quantitation, avidity, and IgM response. J Med Virol 2008;80:95-101.

28. Purdy MA, Khudyakov YE. Evolutionary history and population dynamics of hepatitis E virus. PLoS One 2010;5:e14376.

29. Nakano T, Takahashi K, Pybus OG, Hashimoto N, Kato H, Okano $\mathrm{H}$, et al. New findings regarding the epidemic history and population dynamics of Japan-indigenous genotype 3 hepatitis $\mathrm{E}$ virus inferred by molecular evolution. Liver Int 2012;32:675-688.

30. Berto A, Grierson S, Hakze-van der Honing R, Martelli F, Johne R, Reetz J, et al. Hepatitis E virus in pork liver sausage, France. Emerg Infect Dis 2013;19:264-266.

31. Berto A, Martelli F, Grierson S, Banks M. Hepatitis E virus in pork food chain, United Kingdom, 2009-2010. Emerg Infect Dis 2012;18: 1358-1360.

32. Di Bartolo I, Diez-Valcarce M, Vasickova P, Kralik P, Hernandez M, Angeloni G, et al. Hepatitis E virus in pork production chain in Czech Republic, Italy, and Spain, 2010. Emerg Infect Dis 2012;18:12821289.

33. Colson P, Borentain P, Queyriaux B, Kaba M, Moal V, Gallian P, et al. Pig liver sausage as a source of hepatitis $\mathrm{E}$ virus transmission to humans. J Infect Dis 2010;202:825-834.

34. Chalupa P, Vasickova P, Pavlik I, Holub M. Endemic hepatitis E in the Czech Republic. Clin Infect Dis 2014;58:509-516.

Author names in bold designate shared co-first authorship.

\section{Supporting Information}

Additional Supporting Information may be found in the online version of this article at the publisher's website. 